\title{
Novel Application of Taq DNA Polymerase for Electrochemical Detection of Sexually Transmitted Pathogenic DNA
}

\author{
Hyowon Jang ${ }^{1}$, Hyoyong kim ${ }^{1}$, Seoyoung Lee ${ }^{1}$, Hyun Gyu Park ${ }^{1^{*}}$ \\ ${ }^{1}$ Department of Chemical and Biomolecular Engineering (BK 21+ program), KAIST \\ Daehak-ro 291, Yuseong-gu, Daejeon 3414, South Korea \\ popo987as@kaist.ac.kr; naeggeo2649@kaist.ac.kr \\ eileen93@kaist.ac.kr; hgpark1@kaist.ac.kr
}

\section{Extended Abstract}

We, herein, describe a novel Taq DNA polymerase-induced electrochemical signal amplification reaction capable of detecting sexually transmitted pathogenic DNA by focusing on the Flap endonuclease (FEN) activity of Taq DNA polymerase. In this strategy, we employed specially designed Taqman probe which has 3' overhang region to hold the capture probe and whose 5' end is modified with free thiol to covalently bond with the invertase. In the presence of the target DNA, Taqman probe is subjected to continuously repeated cleavage by the Flap endonuclease (FEN) activity of Taq DNA polymerase, consequently releasing invertase-modified part from intact Taqman probe. After the cleavage reaction, we add capture probe which is already conjugated with magnetic bead and take supernatant of the solution during applying the magnetic force. When we add sucrose into the supernatant taken from previous step, sucrose is hydrolysed to the glucose by enzymatic reaction of invertase, which can be monitored in personal glucose meter (PGM). On the other hand, Taqman probe is not cleaved in the absence of target DNA, which results in the negligible glucose signal from the PGM. To check that the enzymatic cleavage works well as we expected, we prepared various types of samples that contains different dna polymerase respectively, which then the reaction samples were subjected to maldi-tod analysis. As a result, we could confirm that the cleavage of Taq primer only occurs in the presence of the target dna and taq dna polymerase, which means that the taq primer is cleaved by flap endonuclease activity of the taq dna polymerase. After that, we optimized the several reaction conditions such as gox incubation time, magnetic separation time, buffer composition and concentration of each reaction components to clearly discriminate the target sample against the negative sample which does not contain the target dna. Based on this optimal reaction condition, we successfully detected 100 copy of sexually transmitted pathogenic dna which is extracted as form of plasmid dna from cultivated e.coli cell. The novel design principle employed in this work would provide great insight for the development of other biomolecule detection strategy. 